\title{
Integrated optical model for organic light-emitting devices
}

\author{
Saso Mladenovski, ${ }^{1, a)}$ Simone Hofmann, ${ }^{2}$ Sebastian Reineke, ${ }^{2}$ Lieven Penninck, ${ }^{1}$ \\ Thomas Verschueren, ${ }^{1}$ and Kristiaan Neyts ${ }^{1}$ \\ ${ }^{1}$ Electronics and Information Systems Department, Ghent University, Sint-Pietersnieuwstraat 41, B-9000 Gent, \\ Belgium \\ ${ }^{2}$ Institut für Angewandte Photophysik, Technische Universität Dresden, George-Bähr-Str. 1, 01062 Dresden, \\ Germany
}

(Received 27 December 2010; accepted 14 March 2011; published online 26 April 2011)

One of the most important parameters of organic light-emitting devices (OLEDs) in their application for illumination or displays is their efficiency. In order to maximize the efficiency, one needs to understand all loss mechanisms and effects present in these devices and properly model them. For that purpose, we introduce an integrated model for light emission from OLEDs. The model takes into account the exciton decay time change and light outcoupling. Furthermore, it shows how to calculate the external quantum efficiency, the spectral radiance and the luminous current efficacy of OLEDs. The overall theory is experimentally verified through a range of measurements done on a set of green OLED samples with an Ir-based phosphorescent emitter. From the analysis of simulations and experiments one can estimate the charge balance in the OLED stack and the radiative efficiency of the emitter. (C) 2011 American Institute of Physics. [doi:10.1063/1.3576114]

\section{INTRODUCTION}

OLEDs are already commercially available as flat panel displays in a range of portable devices and are already integrated in a variety of artistically designed lamps. The great potential of OLEDs in these two applications is that they may outperform currently available devices on the market. For both applications, the efficiency is one of the key parameters for OLEDs. With the intention to improve the OLED's efficiency, one needs to understand all physical processes in the device, properly model them, and optimize the necessary parameters.

The paper presents an integrated model for all optical effects in planar OLEDs with phosphorescent emitters by including the exciton decay time change and light outcoupling. Furthermore, the model gives the link of the electrooptical effects present in OLEDs through the external quantum efficiency $(E Q E)$ assuming that the electrical losses in the devices stay constant. In addition, one can find the correlation between quantum, radiometric and photometric quantities such as the $E Q E$, the luminous current efficacy ( $L C E)$ and the spectral radiance $(L)$. All the effects predicted by the integrated model are experimentally verified through measurements of the corresponding quantities for a set of green OLED samples. These experiments include measurement of the current-voltage characteristic, exciton decay time, external quantum efficiency, luminous current efficacy and spectral radiance for all samples.

\section{THE INTEGRATED MODEL}

In this section, we introduce the integrated model and explain the different loss mechanisms present in OLEDs.

\footnotetext{
a) Author to whom correspondence should be addressed. Electronic mail: mladsaso@yahoo.com.
}

The main focus of the first part is on the optical effects present in the device: exciton decay time change and light outcoupling. These effects and the correlation between them are explained through the factors that form the external quantum efficiency of the device. The second part of this section gives the relation between the different types of quantities that describe the properties of OLEDs: quantum, radiometric, and photometric quantities like the EQE, the spectral radiance and luminous current efficacy. The whole theory is valid at low excitation levels, before the appearance of bimolecular annihilation ${ }^{1}$ processes.

\section{A. External quantum efficiency (EQE)}

The external quantum efficiency of an OLED is defined as the number of emitted photons $\left(N_{p h}\right)$ divided by the number of injected electrons $\left(N_{e^{-}}\right)$in the device i.e.,

$$
E Q E=\frac{N_{p h}}{N_{e^{-}}} .
$$

There are different loss mechanisms and effects present in an OLED that have different contributions to the EQE. Typically the external quantum efficiency is represented by four different factors, ${ }^{2-5}$ each of them related to a loss mechanism in the OLED:

$$
E Q E=\eta_{c b} \eta_{s t} \eta_{\text {rad }} \eta_{\text {out }}
$$

The first factor is called the charge balance $\eta_{c b}$ and it gives the ratio between the number of excitons formed in the emitting layer and the number of injected electron-hole pairs in the device. It depends on the injection of charges from the electrodes into the organic layers, the conductivity of the organic layers and the different energy levels of the lowest unoccupied molecular orbital (LUMO) and highest occupied 
molecular orbital (HOMO) among the layers. The factor $\eta_{s t}$ gives the fraction of excitons that are in a state that can emit light. Typically, $\eta_{s t}=0.25$ if only singlet states can emit light (fluorescent emitters) and $\eta_{s t}=1$ in the case of phosphorescent emitters ${ }^{2}$ (for guest-host emitting layers all the exctions are transferred on the triplet state of the guest emitting molecule which can emit light). The radiative efficiency $\eta_{\text {rad }}$ gives the percentage of excitons in an emitting state that decay through electromagnetic radiation. The last factor is the outcoupling efficiency $\eta_{\text {out }}$ which gives the fraction of the total generated electromagnetic radiation that manages to escape from the device i.e., the detectable photons.

In this section, we present how to properly model the radiative and outcoupling efficiency for OLEDs with phosphorescent emitters, and furthermore explain the strong correlation between the two factors. For modeling of these two effects we use an ensemble of incoherent electrical dipole antennas with random orientation, ${ }^{6,7}$ used in earlier work. ${ }^{8-11}$ It is assumed that the OLED is a one-dimensional layer structure since its lateral dimensions $(>1 \mathrm{~mm})$ are much larger than its thickness $(\sim 1 \mu \mathrm{m})$. The emission of the dipole antenna is decomposed in plane and evanescent coherent waves with a transverse electric (TE) and transverse magnetic (TM) polarization. The model takes into account wide-angle and multiple-beam interference caused by partial reflection, total internal reflection and absorption. It is assumed that the emitting layer in the OLED is nonabsorbing, which is often the case for the wavelength region in which the emission occurs.

The radiative efficiency $\eta_{\text {rad }}$ of an emitting organic material is directly related to the exciton decay time $\tau$. It is well known $n^{3,10,12}$ that the exciton decay time depends on the optical environment (the layer thicknesses and refractive indices of the materials in the OLED) which is equivalent to the Purcell effect. ${ }^{13}$ Therefore, $\eta_{\text {rad }}$ also depends on the optical environment. For the effect of exciton decay time change we use our previously verified model m $^{3,12,14}$ for OLEDs with phosphorescent emitters, that is founded on the emission of a dipole antenna ensemble. For this type of emitters, the exciton decays from a triplet state of the phosphorescent molecule onto the ground state. Our model assumes that the exciton can decay through two channels: a radiative and a nonradiative one. Which decay channel will the exciton follow depends on the probabilities of the two processes, namely their decay rates $\Gamma_{r}$ and $\Gamma_{n r}$. It is assumed that the probability for a radiative decay is proportional to the total generated power by the antenna ensemble.

As a reference, we use an antenna ensemble in an infinite homogeneous medium with the same refractive index as the emitting layer (EML). In this case, the inverse value of the decay time i.e. the total decay rate is given by: $:^{3,12}$

$$
\frac{1}{\tau_{0}}=\Gamma_{0}=\Gamma_{r, 0}+\Gamma_{n r, 0},
$$

where $\Gamma_{r, 0}$ and $\Gamma_{n r, 0}$ are the radiative and nonradiative decay rates in an infinite medium. From here, the radiative efficiency in an infinite medium for a specific emitter is calculated as:

$$
\eta_{\text {rad }, 0}=\frac{\Gamma_{r, 0}}{\Gamma_{r, 0}+\Gamma_{n r, 0}} .
$$

If the EML is placed in a layer stack such as the OLED, then $\Gamma_{r, 0}$ is modified by a factor $F$. Therefore Eqs. (3) and (4) for an EML in an OLED are written as:

$$
\frac{1}{\tau}=\Gamma=F \Gamma_{r, 0}+\Gamma_{n r, 0}
$$

and

$$
\eta_{\text {rad }}=\frac{F \Gamma_{r, 0}}{F \Gamma_{r, 0}+\Gamma_{n r, 0}}
$$

where $\tau, \Gamma$, and $\eta_{\text {rad }}$ are the exciton decay time, exciton decay rate and the radiative efficiency of the emitter in the layer stack. The modification factor $F$ is the total power generated by the dipole antenna ensemble, divided by the power of the same ensemble in an infinite medium. Therefore in the case of an infinite medium $F=1$, and Eqs. (5) and (6) become equivalent to Eqs. (3) and (4). The model assumes that the width of the emission spectrum is due to a homogeneous broadening of the triplet state of the emitting guest molecule. This means that the radiative decay of an exciton is described by an ensemble of electrical dipole antennas with random orientation, each of them with the same spectral distribution. The dipole antennas have a random orientation due to the symmetrical orientation of the small emitting molecules and the exciton hopping among the molecules. For that reason, all the excitons have the same value of $F$, which leads to a pure exponential decay. This monoexponential decay can be seen in the measurements of decay signals for Ir-based emitters at low-excitation intensities reported previously. ${ }^{1,12}$

In the case of homogeneous broadening of the excited state with a normalized spectral density $S_{0}(\lambda)$ in an infinite homogeneous medium $\int_{0}^{\infty} S_{0}(\lambda) d \lambda=1$, the total generated power $F$, which determines the change in the radiative decay, is calculated by weighing the dipole antenna emission with the spectral density:

$$
F=\int_{0}^{\infty} S_{0}(\lambda) \mathrm{d} \lambda \int_{0}^{\infty} K_{E M L}(\kappa, \lambda) \mathrm{d} \kappa^{2}
$$

In the formula above, $K_{E M L}(\kappa, \lambda)=K_{E M L}^{+}(\kappa, \lambda)+K_{E M L}^{-}(\kappa, \lambda)$ where $K_{E M L}^{+}$and $K_{E M L}^{-}$are the power densities per interval $\mathrm{d} \kappa^{2}=2 \kappa \mathrm{d} \kappa$ through a plane in the emitting layer (EML) toward the anode and the cathode respectively, ${ }^{7}$ emitted by an ensemble of randomly oriented dipole antennas placed in the middle of the EML. The parameter $\kappa$ is the length of the projection of the spatial frequency of the plane or evanescent wave on a surface parallel to the layer structure. Placing the dipole antennas in the middle of the EML is a good approximation even though the location of the exciton decay will depend on the ambipolar character of the EML. This approximation is particularly appropriate because of the typically thin EML $(\leq 20 \mathrm{~nm})$ used in OLEDs with small molecule emitters. 
As explained above, the outcoupling efficiency $\eta_{\text {out }}$ gives the fraction of the total generated electromagnetic radiation that manages to escape from the device i.e., the detectable photons. Having in mind that the OLED is a thin film layer structure (thickness $\sim 1 \mu \mathrm{m}$ ) deposited usually on a thick glass substrate (thickness $\sim 1 \mathrm{~mm}$ ), the outcoupling efficiency can be split in two parts: outcoupling from OLED to substrate $\left(\eta_{O L E D, s u b}\right)$ and outcoupling from substrate to air $\left(\eta_{\text {sub,air }}\right)$, and written as:

$$
\eta_{\text {out }}=\eta_{O L E D, s u b} \eta_{\text {sub,air }}
$$

The main reason for this is that the OLED to substrate emission comes from a thin-film layer structure with thicknesses of the order of the wavelength of visible light. Therefore, this emission needs to be modeled using coherent light (Fig. 1). On the other hand, the outcoupling from substrate into air needs to be modeled by incoherent light because the substrate has a thickness much larger than the light wavelength.

We model the OLED to substrate emission using an ensemble of incoherent electrical dipole antennas with random orientation just as in the case of exciton decay time change. The OLED to substrate outcoupling efficiency is defined as:

$$
\eta_{O L E D, s u b}=\frac{I_{s u b}}{F}
$$

where $F$ is calculated by Eq. (7) and:

$$
I_{\text {sub }}=\int_{0}^{\infty} S_{0}(\lambda) \mathrm{d} \lambda \int_{0}^{2 \pi n_{\text {sub }} / \lambda} K_{\text {sub }}(\kappa, \lambda) \mathrm{d} \kappa^{2}
$$

gives the total power emitted in the substrate with refractive index $n_{s u b}$, where $K_{s u b}$ is the power density per interval $\mathrm{d} \kappa^{2}=2 \kappa d \kappa$ through a plane in the substrate emitted by an ensemble of randomly oriented dipole antennas placed in the middle of the EML. The parameter $\kappa$ is the projection of the spatial frequency (the wave vector) of the plane wave, on the plane parallel to the layer structure. ${ }^{6,7}$ Namely,

$$
\kappa=\frac{2 \pi n_{\text {sub }}}{\lambda} \sin \theta_{\text {sub }}
$$

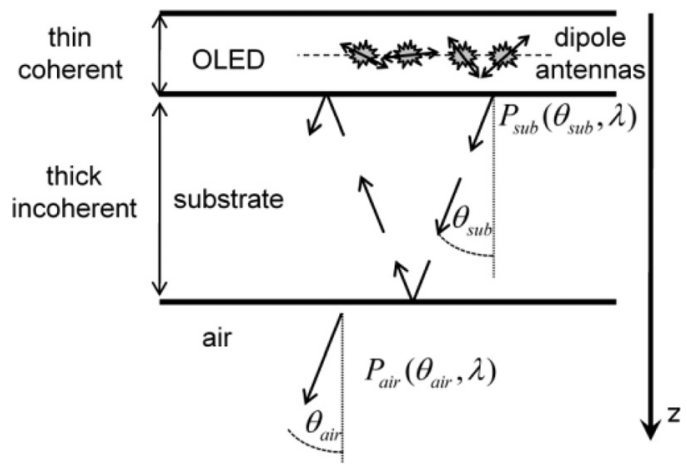

FIG. 1. Simplified optical structure of an OLED showing the coherent and incoherent part of the integrated model. $P_{\text {air }}$ and $P_{\text {sub }}$ are the simulated powers per unit solid angle in air and substrate as function of the wavelength $\lambda$ and the inclination angles $\theta_{\text {air }}$ and $\theta_{\text {sub }}$, correspondingly. and it can obtain values in the range of

$$
0<\kappa<\frac{2 \pi n_{\text {sub }}}{\lambda}
$$

Having this in mind, we can replace $K_{\text {sub }}$ by a power density per unit solid angle $\left(\Omega_{\text {sub }}\right)$ in the substrate ${ }^{6} P_{\text {sub }}\left(\theta_{\text {sub }}, \lambda\right)$ which depends only on the inclination angle $\theta_{\text {sub }}$, and not on the azimuth angle $\varphi_{\text {sub }}$ due to the rotational symmetry of the layer structure. The relation between the two power densities is given by:

$$
\int_{0}^{2 \pi n_{\text {sub }} / \lambda} K_{\text {sub }}(\kappa, \lambda) \mathrm{d} \kappa^{2}=\iint_{2 \pi} P_{\text {sub }}\left(\theta_{\text {sub }}, \lambda\right) d \Omega_{\text {sub }} .
$$

From Eq. (11) above and Eq. (10), follows that the total power emitted in the substrate can be calculated as:

$$
\begin{aligned}
I_{\text {sub }} & =\int_{0}^{\infty} S_{0}(\lambda) \mathrm{d} \lambda \iint_{2 \pi} P_{\text {sub }}\left(\theta_{\text {sub }}, \lambda\right) d \Omega_{\text {sub }} \\
& =2 \pi \int_{0}^{\infty} S_{0}(\lambda) \mathrm{d} \lambda \int_{0}^{\pi} P_{\text {sub }}\left(\theta_{\text {sub }}, \lambda\right) \sin \theta_{\text {sub }} d \theta_{\text {sub }} .
\end{aligned}
$$

Using Eqs (9), (7) and (12), one can calculate the outcoupling efficiency from OLED into the substrate $\eta_{O L E D, s u b}$.

To model the outcoupling from the substrate into air, one needs to use incoherent light since the thickness of the substrate is much larger than the wavelength of visible light. Due to the partial reflection at the substrate/air interface and the reflection from the OLED, one needs to take into account all multiple reflections of light inside the substrate for a precise calculation of light outcoupling from substrate into air. In the case of a planar substrate and a planar OLED (i.e., no outcoupling structures) both interfaces of the substrate are nondepolarizing. This means that there is no coupling of light with polarization TM into polarization TE and vice versa. Therefore, we determine the light outcoupling from the substrate into air by summing up the separately calculated outcoupled power densities for TM and TE polarization. The reflectivity and transmission of the substrate/OLED interface and substrate/air interface are calculated individually for each polarization. With this approach, using the already known values for $P_{\text {sub }}\left(\theta_{\text {sub }}, \lambda\right)$ and Snell's law, one can easily calculate the power per unit solid angle in air $P_{\text {air }}\left(\theta_{\text {air }}, \lambda\right)$. From here one can determine the total power that is emitted into air:

$$
\begin{aligned}
I_{\text {air }} & =\int_{0}^{\infty} S_{0}(\lambda) \mathrm{d} \lambda \iint_{2 \pi} P_{\text {air }}\left(\theta_{\text {air }}, \lambda\right) d \Omega_{\text {air }} \\
& =2 \pi \int_{0}^{\infty} S_{0}(\lambda) \mathrm{d} \lambda \int_{0}^{\pi} P_{\text {air }}\left(\theta_{\text {air }}, \lambda\right) \sin \theta_{\text {air }} d \theta_{\text {air }} .
\end{aligned}
$$

The outcoupling efficiency from substrate into air is calculated as the following ratio:

$$
\eta_{\text {sub }, \text { air }}=\frac{I_{\text {air }}}{I_{\text {sub }}}
$$


where $I_{s u b}$ and $I_{\text {air }}$ are the total power emitted in the substrate and air respectively and are calculated using Eqs. (12) and (13).

Using the expressions for $\eta_{O L E D, s u b}$ and $\eta_{\text {sub,air }}$ given in Eqs. (9) and (14) one can calculate the total outcoupling efficiency:

$$
\eta_{\text {out }}=\frac{I_{\text {air }}}{F}
$$

In summary, we provided a model for the radiative efficiency $\eta_{\text {rad }}$, the OLED to substrate $\eta_{O L E D, s u b}$ and the substrate to air $\eta_{\text {sub.air }}$ outcoupling efficiencies. Having in mind that both $\eta_{\text {rad }}$ and $\eta_{O L E D, \text { sub }}$ are calculated using the same model of an antenna ensemble with coherent light, and both of them contain the total generated power $F$, it is evident that these two factors should be calculated together. Taking all this into account and using Eqs. (2), (6) and (15), we can write down the expression for EQE in air for OLEDs with phosphorescent emitters $\left(\eta_{s t}=1\right)$ :

$$
E Q E_{\text {air }}=\eta_{c b} \frac{I_{a i r} \Gamma_{r, 0}}{F \Gamma_{r, 0}+\Gamma_{n r, 0}},
$$

which gives the ratio of the number of photons emitted into air over the number of electrons injected in the OLED. In the same manner one can define an EQE in the substrate:

$$
E Q E_{\text {sub }}=\eta_{c b} \frac{I_{s u b} \Gamma_{r, 0}}{F \Gamma_{r, 0}+\Gamma_{n r, 0}}
$$

which gives the ratio of the number of photons emitted in the substrate over the number of electrons injected in the OLED. The number of photons emitted into the substrate can be measured by using a large half-ball lens attached on the substrate, and by placing the OLED in the center of the lens. Obviously, it is important in this configuration that the halfball lens is much larger than the OLED.

Once we are able to properly calculate the quantum quantities (like the EQE), the main challenge is to relate them with radiometric and photometric quantities like the spectral radiance and luminous current efficacy.

\section{B. Spectral radiance and luminous current efficacy}

In this section we describe a model on how one can calculate the spectral radiance $L(\theta, \lambda, j)$ as a function of the inclination angle $\theta$, the light wavelength $\lambda$ and the current density $j$. The $L(\theta, \lambda, j)$ gives the power of the emitted light per unit projected area (perpendicular to the viewing direction $\theta$ ), per unit solid angle, per unit wavelength and is expressed in

$$
\left[\frac{\mathrm{W}}{\mathrm{m}^{2} \cdot \mathrm{sr} \cdot \mathrm{nm}}\right]
$$

Afterwards we determine how to calculate the luminous current efficacy (LCE). The LCE gives the total emitted luminous flux by the device per unit input current and is expressed in

$$
\left[\frac{\operatorname{lm}}{\mathrm{A}}\right] \text {. }
$$

The efficiency should be constant for low current densities, before bimolecular annihilation processes appear. ${ }^{1}$

First we will derive a formula to calculate the spectral radiance $L(\theta, \lambda, j)$ of an OLED. An injected electron in the OLED has an elementary charge $e=1.6 \times 10^{-19} \mathrm{C}$ and can lead to an emission of a photon with energy $h c / \lambda$, where $h=6.626 \times 10^{-34} \mathrm{~m}^{2} \cdot \mathrm{kg} / \mathrm{s}$ is Planck's constant and $c=3 \times 10^{8} \mathrm{~m} / \mathrm{s}$ is the speed of light in vacuum. The ratio between the energy of the emitted photon and the injected elementary charge is given by $h c / \lambda e$. However, due to the loss mechanisms described previously, not every injected electron will lead to the emission of a photon. From the previous section on the EQE, one can determine the probability for the emission of a photon in air with a wavelength $\lambda$ in a direction $\theta$ per unit wavelength, per unit solid angle and per injected electron:

$$
\rho_{\text {air }}(\theta, \lambda)=\eta_{c b} \frac{\Gamma_{r, 0}}{F \Gamma_{r, 0}+\Gamma_{n r, 0}} S_{0}(\lambda) P_{\text {air }}\left(\theta_{\text {air }}, \lambda\right) .
$$

The probability $\rho_{\text {air }}\left(\theta_{\text {air }}, \lambda\right)$ is expressed in $[1 /(\mathrm{nm} \cdot \mathrm{sr})]$ and is directly related to the EQE in air [see Eq. 16]:

$$
E Q E_{\text {air }}=\int_{0}^{\infty} \iint_{2 \pi} \rho_{\text {air }}\left(\theta_{\text {air }}, \lambda\right) d \Omega_{\text {air }} d \lambda .
$$

For low values of $j$, one can assume that $L(\theta, \lambda, j)$ is proportional to $j$. Using all the above outlined factors we write down the following expression for the spectral radiance in air:

$$
L_{\text {air }}\left(\theta_{\text {air }}, \lambda, j\right)=\frac{h c}{\lambda e} j \frac{1}{\cos \theta_{\text {air }}} \rho_{\text {air }}\left(\theta_{\text {air }}, \lambda\right)
$$

The factor $1 / \cos \theta_{\text {air }}$ is needed since $L(\theta, \lambda, j)$ is per unit projected area. Using Eq. (18), the above formula for the spectral radiance can be written as:

$$
L_{\text {air }}\left(\theta_{\text {air }}, \lambda, j\right)=\frac{h c}{\lambda e} \eta_{c b} j \frac{\Gamma_{r, 0}}{F \Gamma_{r, 0}+\Gamma_{n r, 0}} \frac{1}{\cos \theta_{\text {air }}} S_{0}(\lambda) P_{\text {air }}\left(\theta_{\text {air }}, \lambda\right)
$$

Equation (21) gives the spectral radiance for the light emitted into air. In an equivalent way as for the EQE in substrate, one can define a spectral radiance for the light emitted in the substrate:

$$
\begin{aligned}
L_{\text {sub }}\left(\theta_{\text {sub }}, \lambda, j\right)= & \frac{h c}{\lambda e} \eta_{c b} j \frac{\Gamma_{r, 0}}{F \Gamma_{r, 0}+\Gamma_{n r, 0}} \frac{1}{\cos \theta_{\text {sub }}} \\
& \times S_{0}(\lambda) P_{\text {sub }}\left(\theta_{\text {sub }}, \lambda\right) .
\end{aligned}
$$

One can derive a formula for the luminous current efficacy ( $L C E$ ) which gives the total emitted luminous flux per unit input current in the OLED. For this purpose one needs to weight $L(\theta, \lambda, j)$ with the eye sensitivity curve $V(\lambda)$ (maximum set to one), integrate over the whole spectral density 
$S_{0}(\lambda)$ and solid angle $\Omega$, and divide by the input current density $j$. The luminous current efficacy in air is calculated as:

$$
L C E_{\text {air }}=\frac{683 \frac{\operatorname{lm}}{\mathrm{W}} \int_{0}^{\infty} V(\lambda) \iint_{2 \pi} L_{\text {air }}\left(\theta_{\text {air }}, \lambda, j\right) \cos \theta_{\text {air }} d \Omega_{\text {air }} d \lambda}{j} .
$$

The factor $\cos \theta_{\text {air }}$ is needed to return to the unit active area on the device. Using Eq. (21) we can write:

$$
\begin{aligned}
L C E_{\text {air }}= & 683 \frac{\mathrm{lm}}{\mathrm{W}} \cdot \eta_{c b} \int_{0}^{\infty} V(\lambda) \iint_{2 \pi} \frac{h c}{\lambda e} \\
& \times \frac{\Gamma_{r, 0}}{F \Gamma_{r, 0}+\Gamma_{n r, 0}} S_{0}(\lambda) P_{\text {air }}\left(\theta_{\text {air }}, \lambda\right) d \Omega_{\text {air }} d \lambda
\end{aligned}
$$

In a comparable way, one can also define a luminous current efficacy in the substrate:

$$
\begin{aligned}
L C E_{\text {sub }}= & 683 \frac{\mathrm{lm}}{\mathrm{W}} \cdot \eta_{c b} \int_{0}^{\infty} V(\lambda) \iint_{2 \pi} \frac{h c}{\lambda e} \\
& \times \frac{\Gamma_{r, 0}}{F \Gamma_{r, 0}+\Gamma_{n r, 0}} S_{0}(\lambda) P_{s u b}\left(\theta_{s u b}, \lambda\right) d \Omega_{s u b} d \lambda .
\end{aligned}
$$

The $L C E$ is a well defined quantity for OLEDs that can be calculated with the presented formulas and measured more accurately, compared to the usually used luminous power efficacy (expressed in $[\mathrm{lm} / W]$ ) which gives the total emitted luminous flux per unit input power. The luminous power efficacy depends on the voltage. In order to have good estimations of the voltage one needs to know the electrical parameters of the organic layers, which is not the subject of this work. Furthermore, in experiments can be difficult to estimate the voltage especially if there is an important voltage drop over the electrode lines.

With the formulas above, we have built an integrated model that allows us to calculate the $E Q E$, the spectral radiance $L(\theta, \lambda, j)$ and $L C E$ of an OLED at lower current densities. In the formulas, besides the optical effects, the following parameters determine the behavior of the device: the charge balance $\eta_{c b}$, and the radiative $\Gamma_{r, 0}$ and nonradiative $\Gamma_{n r, 0}$ decay rates of the emitter in an infinite homogeneous medium. These parameters can be estimated by fitting the simulations and measurements of decay times and external quantum efficiencies. The experiments for determining these parameters and for verification of our integrated model are presented in the following sections.

\section{DEVICE FABRICATION AND MEASUREMENTS}

In order to test the integrated model, we fabricated a set of green OLED samples (Fig. 2). The organic layers are sandwiched between a $90 \mathrm{~nm}$ transparent indium tin oxide (ITO) anode and a highly reflecting aluminum (Al) cathode of $100 \mathrm{~nm}$. The OLED architecture contains doped charge transport layers and intrinsic charge blocking layers. ${ }^{15,16}$ We employ $4 \mathrm{wt} \%$ of NDP-2 (Novaled AG) doped in the NHT-5 (Novaled AG) as hole injection and transport layer (HTL). As electron and hole blocking layers (EBL/HBL), we

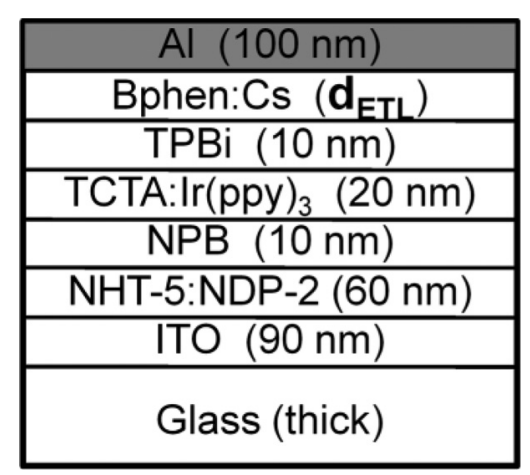

FIG. 2. Device structure of the fabricated OLED samples illustrating the abbreviations of the used materials and the layer thicknesses. $d_{\text {ETL }}$ is the layer thickness of the electron-transport layer (BPhen:Cs) which is a varying parameter in the fabricated OLEDs and in the simulations.

use $10 \mathrm{~nm}$ of $\mathrm{N}, \mathrm{N}^{\prime}$-Di(naphthalen-1-yl)-N, $\mathrm{N}^{\prime}$-diphenyl-benzidine (NPB) and 2,2',2' -(1,3,5-Phenylen)tris(1-phenyl-1Hbenzimidazol) (TPBi), respectively. The electron injection and transport layer (ETL) is realized by a 4,7-diphenyl-1,10phenanthroline (BPhen) layer doped with Cesium (Cs). We fabricated four devices with a $20 \mathrm{~nm}, 45 \mathrm{~nm}, 130 \mathrm{~nm}$, and $210 \mathrm{~nm}$ thickness of BPhen:Cs, respectively. The emitting layer (EML) is a host-guest-system, where 4,4',4' '-tris(carbazol-9-yl)-triphenylamine (TCTA) acts as matrix material for the phosphorescent emitter tris(2-phenylpyridin) iridium(III) $\left(\operatorname{Ir}(\text { ppy })_{3}\right)$, which is doped with $8 \mathrm{wt} \%$.

All devices are fabricated by thermal evaporation on ITO prestructured glass substrates in an UHV chamber (Kurt J. Lesker) with a base pressure below $10^{-7}$ mbar. Furthermore, the OLEDs were encapsulated immediately after preparation under nitrogen atmosphere using glass lids and epoxy glue. The size of the rectangular (close to square looking) active area is $6.7 \mathrm{~mm}^{2}$.

Transient electroluminescence (EL) experiments are carried out to determine the exciton decay time of the phosphorescent emitter within a given optical environment. The OLED devices are electrically excited using a $30 \mu$ s voltage pulse with a frequency of $100 \mathrm{~Hz}$ generated by a Hewlett Packard 8114A pulse generator. The emitted light is collected with a fast photodiode PDA10A-EC (Thorlabs), which is connected to a multichannel oscilloscope. ${ }^{17}$ In order to realize monoexponential decay by excluding the influence of triplet-triplet annihilation (TTA) ${ }^{1}$ at high excitation levels, the excitation voltage is adjusted to low levels (corresponding current densities $<4 \mathrm{~A} / \mathrm{m}^{2}$ ), where the TTA contribution is weak.

Figure 3 shows the EL transients of all four OLEDs having different ETL thickness. As expected from the low excitation level, the decay curves follow monoexponential decay directly after the end of the voltage pulse. However, in contrast to a fully monoexponential behavior, a second component with a longer time constant is observed at the long-lived tail of the decay (cf. inset of Fig. 3). Being observed for all devices, it is concluded that this delayed component is an extrinsic (not representing the nature of the emitter) feature of the OLEDs prepared and is either due to an energy backtransfer in the EML ${ }^{18}$ or due to delayed carrier recombination. ${ }^{17,19}$ Thus, in order to extract the exciton decay time (the 




FIG. 3. (Color online) Measured electroluminescent (EL) transient signals (dots) for the OLEDs with different ETL thicknesses. Biexponential curves are fitted (lines) of which the fast decay component gives the decay time of the triplet state of $\operatorname{Ir}(\text { ppy })_{3}$ in the OLEDs with different ETL thicknesses (inset axis with same units).

time constant of the faster component in these transients), a biexponential decay law is applied to fit these curves: ${ }^{20}$

$$
I(t)=A \exp (-t / \tau)+B \exp \left(-t / \tau_{\text {delayed }}\right)
$$

where $A$ and $B$ are weighting factors, $\tau$ is the exciton decay time and $\tau_{\text {delayed }}$ is the time constant describing the second component. The resulting calculated fits are plotted in Fig. 3 as solid lines. All transients are consistently fitted with fixed values for $A=0.87$ and $B=0.13$, respectively. Slight variations of the time constant with a value of $(2.00 \pm 0.25 \mu \mathrm{s})$ for the second component are necessary to obtain these fits (possibly because the signal itself is close to the detector noise level and thus sensitive to the subtraction of the signal baseline). Being longer than typical values of the $\operatorname{Ir}(\mathrm{ppy})_{3}$ exciton lifetime (in even weaker cavities) ${ }^{1}$ this supports the assumption to be an extrinsic effect. The corresponding exciton decay times as a result of this fit is given in Fig. 3, ranging from 580 to $850 \mathrm{~ns}$, depending on the ETL thickness.

The Current-Voltage (jV) characteristics shown on Fig. 4 are measured by a source measure unit SMU 2400 (Keithley). The spectral radiance is measured at a forward luminance of $1000 \mathrm{~cd} / \mathrm{m}^{2}$ (i.e., different current densities) for all OLEDs, using a self calibrated spectro-goniometer including a miniature USB4000 fiber optic spectrometer (Ocean Optics). The same measurement system allows us to determine the EQE for different current densities. ${ }^{21}$ Figure 5 shows the dependence of the external quantum efficiency from the current density which is derived by the assumption that the angular distribution of emitted light does not change as function of the current density. This is reasonable, because in a device with one emitter, the position of the emission zone is independent of the current and the optical properties of the device are not affected. ${ }^{21}$

In order to determine the LCE and the EQE in the substrate, all samples are measured in a calibrated Ulbricht Sphere with covered edges and an attached glass half ball

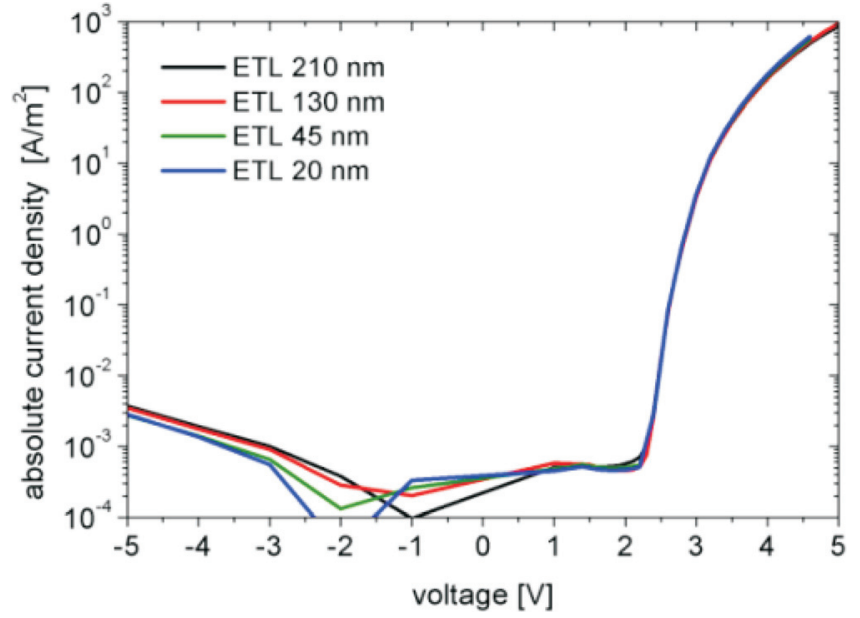

FIG. 4. (Color online) Measured current-voltage characteristics of the OLEDs with different ETL thickness.

lens (Biomedical Optics, $18 \mathrm{~mm}$ diameter) using refractive index matching oil. The LCE is calculated from the measured luminous power efficacy (LE) $[\mathrm{lm} / \mathrm{W}]$ by multiplication with the voltage.

\section{VERIFICATION OF THE MODEL AND DISCUSSION}

In this section, we present a detailed verification of the integrated model by comparing measurements and simulations, as described in the previous sections. First we start with a confirmation of the exciton decay time model, which has been tested also in previous work. ${ }^{12}$ Eq. (5) predicts that there should be a linear dependency between the inverse value of the measured exciton decay time $\tau$ and the simulated total generated power $F$ by the antenna ensemble. On Fig. 6, one can see that this dependency is indeed linear, and from the linear fit one can estimate the radiative and nonradiative decay rates in an infinite medium for the $\operatorname{Ir}(p p y)_{3}$ emitter i.e., $\Gamma_{r, 0}=0.975 \mu \mathrm{s}^{-1}$ and $\Gamma_{n r, 0}=0.234 \mu \mathrm{s}^{-1}$, respectively. Furthermore, using Eq. (4) one can also estimate the radiative efficiency $\eta_{\text {rad }, 0}=0.81$ for this green emitter in an infinite medium. This means that if the emitters are placed in an infinite nonabsorbing homogeneous EML medium, about $81 \%$ of the formed excitons will decay radiatively. Since, in

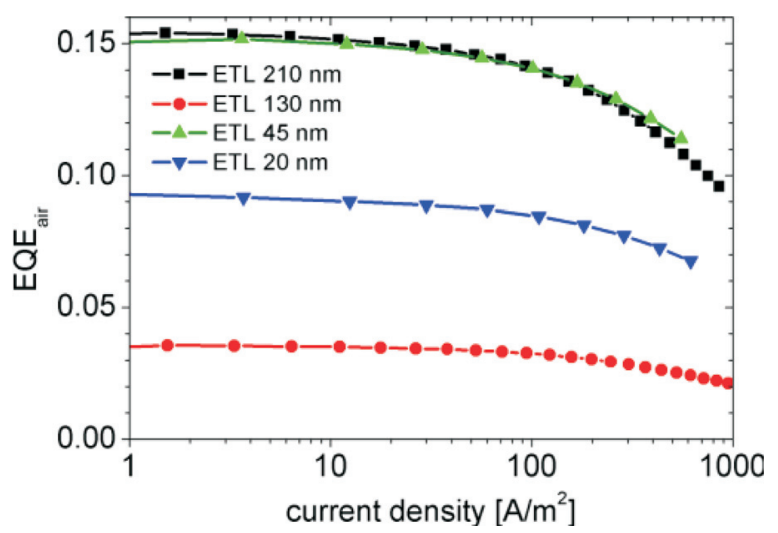

FIG. 5. (Color online) Measured external quantum efficiency in air ( $\left.\mathrm{EQE}_{\text {air }}\right)$ without a half-ball lens as function of the current density. 


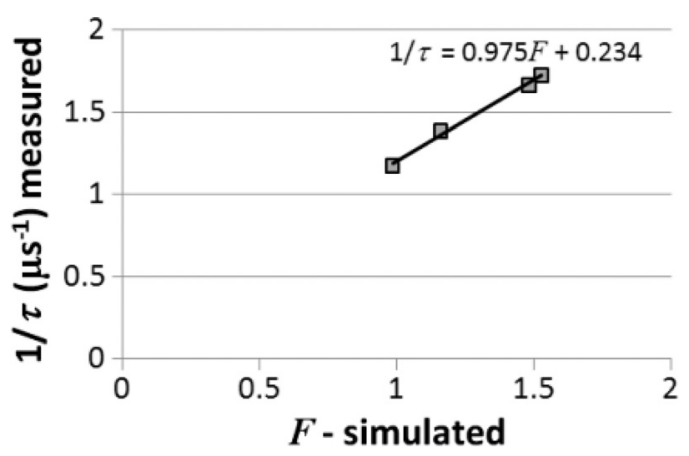

FIG. 6. Measured inverse value of the decay time $1 / \tau$ as function of simulated total generated power $F$ (square points). Linear least square fit (solid line) according to Eq. (5) with the fitted parameters giving the radiative $\Gamma_{\mathrm{r}, 0}=0.975 \mu \mathrm{s}^{-1}$ and nonradiative $\Gamma_{\mathrm{nr}, 0}=0.234 \mu \mathrm{s}^{-1}$ decay rates.

such a nonabsorbing medium there will be no power from the dipole antennas coupled to the evanescent waves, all the radiatively decaying excitons will emit a photon. To further check the exciton decay time model, we also look at the dependency of $F$ and $1 / \tau$ on the ETL thickness $d_{\text {ETL. This is }}$ shown on Fig. 7, where the two vertical axes for $F$ and $1 / \tau$ are properly scaled, taking into account the previously estimated values of $\Gamma_{r, 0}$ and $\Gamma_{n r, 0}$. One can see that the model describes very well the change of the exciton decay time as a function of the ETL thickness.

The next step is the experimental verification of the model for external quantum efficiency (EQE). From Eq. (16), one can see that the only left unknown parameter is the charge balance $\eta_{c b}$. In our model, we assume that the charge balance stays constant when changing the thickness of the ETL layer $d_{\mathrm{ETL}}$. This assumption is based on the measured $j$ $V$ characteristics which are practically identical for all OLED samples (Fig. 4). The grounds for achieving very similar electrical properties of the OLED samples is to use doped ETL and HTL layers and a $p-i-n$ OLED structure. ${ }^{15,16}$ Having this in mind, one can determine $\eta_{c b}$ as a constant fitting parameter by comparing measured EQE values (without a half-ball lens) and simulated $E Q E_{\text {air }}$ values using Eq. (16). The values for the measured EQE are taken at a low current density before the efficiency roll-off sets in (Fig. 5). Such a

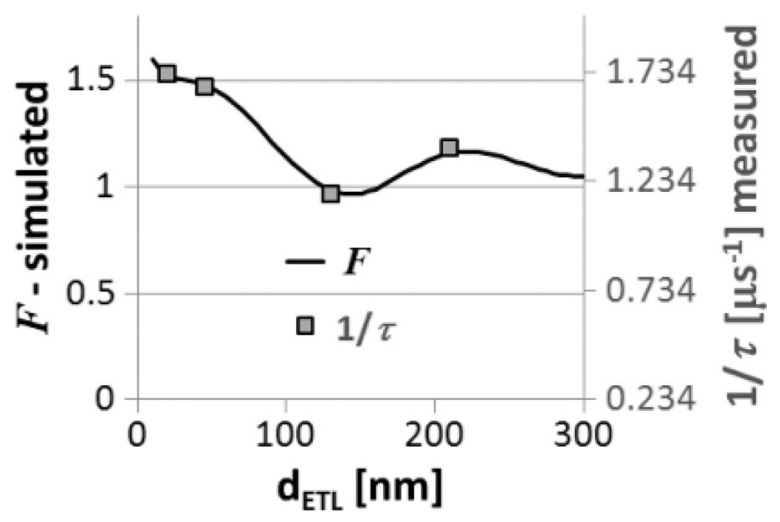

FIG. 7. Simulated total generated power $F$ as function of the electron-transport layer thickness $d_{\mathrm{ETL}}$ (solid line). Measured inverse value of the decay time $1 / \tau$ as function of $d_{\text {ETL }}$ (square points). The right vertical axes $1 / \tau$ is scaled according to Eq. (5) using the values for radiative $\Gamma_{\mathrm{r}, 0}=0.975 \mu \mathrm{s}^{-1}$ and nonradiative $\Gamma_{\mathrm{nr}, 0}=0.234 \mu \mathrm{s}^{-1}$ decay rates.

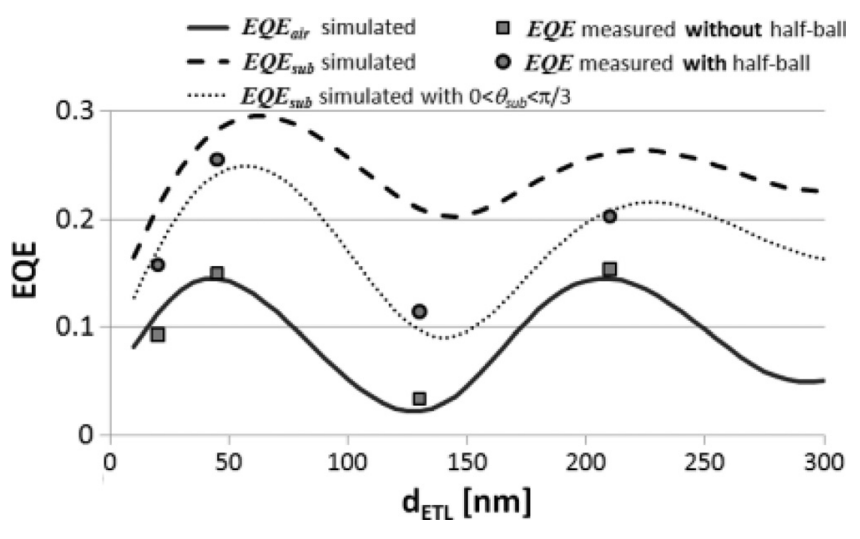

FIG. 8. Simulated external quantum efficiencies in air and substrate $\left(E Q E_{\text {air }}\right.$ and $E Q E_{\text {sub }}$ ) as function of the electron-transport layer thickness $d_{\mathrm{ETL}}$ (solid and dashed line). Simulated $E Q E_{\text {sub }}$ by integrating the inclination angle only in the range $0<\theta_{\text {sub }}<\pi / 3$ (dotted line). Measured EQE without and with an attached half-ball lens on the fabricated OLEDs (squares and circles).

comparison for our OLED samples is shown in Fig. 8, where we give the measured EQE without a half-ball lens (squares) and simulated $E Q E_{\text {air }}$ (solid line) and the estimated charge balance from this fit is $\eta_{c b}=0.80$. Using this value for the charge balance and Eq. (17) we can simulate the $E Q E_{\text {sub }}$ (dashed line on Fig. 8) and compare it with the measurements of the EQE done with a half-ball lens attached (circle points on Fig. 8). In principle, if the half-ball lens is large enough, almost all the photons emitted in the substrate can be outcoupled and detected. However, one can see that the measurements of $E Q E_{s u b}$ with an attached half-ball lens show smaller values then the simulations for $E Q E_{s u b}$. The main reason for this is that it is rather difficult to outcouple all the photons emitted in the substrate and detect them. In our measurements we use a half-ball lens with a diameter of $18 \mathrm{~mm}$, which is not sufficiently large and some total internal reflection occurs. In addition the edges of the substrate are covered by the sample holder. This means that light emitted at higher angles $\theta_{\text {sub }}$ in the substrate is less outcoupled than light emitted at smaller angles. The trapped light in the substrate may be absorbed by the electrode or may reach the edges of the substrate and be absorbed by the sample holder. In order to check this, we calculated $E Q E_{s u b}$ by integrating the inclination angle in a limited range $0<\theta_{\text {sub }}<\pi / 3$ (instead of $0<\theta_{\text {sub }}<\pi / 2$ ). These values are shown on Fig. 8 (dotted line) and fit very well with the measured $E Q E_{\text {sub }}$ with a halfball lens (circles). This indicates that part of the light emitted at higher angles is indeed not well outcoupled by the halfball lens.

The next step is to test the theory for spectral radiance $L_{\text {air }}(\theta, \lambda, j)$. We simulate the spectral radiance in air using Eq. (21) and the current density $j$ at which the measurements without a half-ball lens were done for each specific sample. In general, $L_{\text {air }}(\theta, \lambda, j)$ is measured at low current densities $\left(j \sim 10 \mathrm{~A} / \mathrm{m}^{2}\right)$ for all samples except the OLED with $d_{\mathrm{ETL}}=130 \mathrm{~nm}$ which is measured at $j=304 \mathrm{~A} / \mathrm{m}^{2}$. The main reason for this exception is the low emission from the sample with $d_{\mathrm{ETL}}=130 \mathrm{~nm}$ since it is in the minimum of the EQE (see Fig. 8). The simulations and measurements of the spectral radiance together with the current densities, at which the 

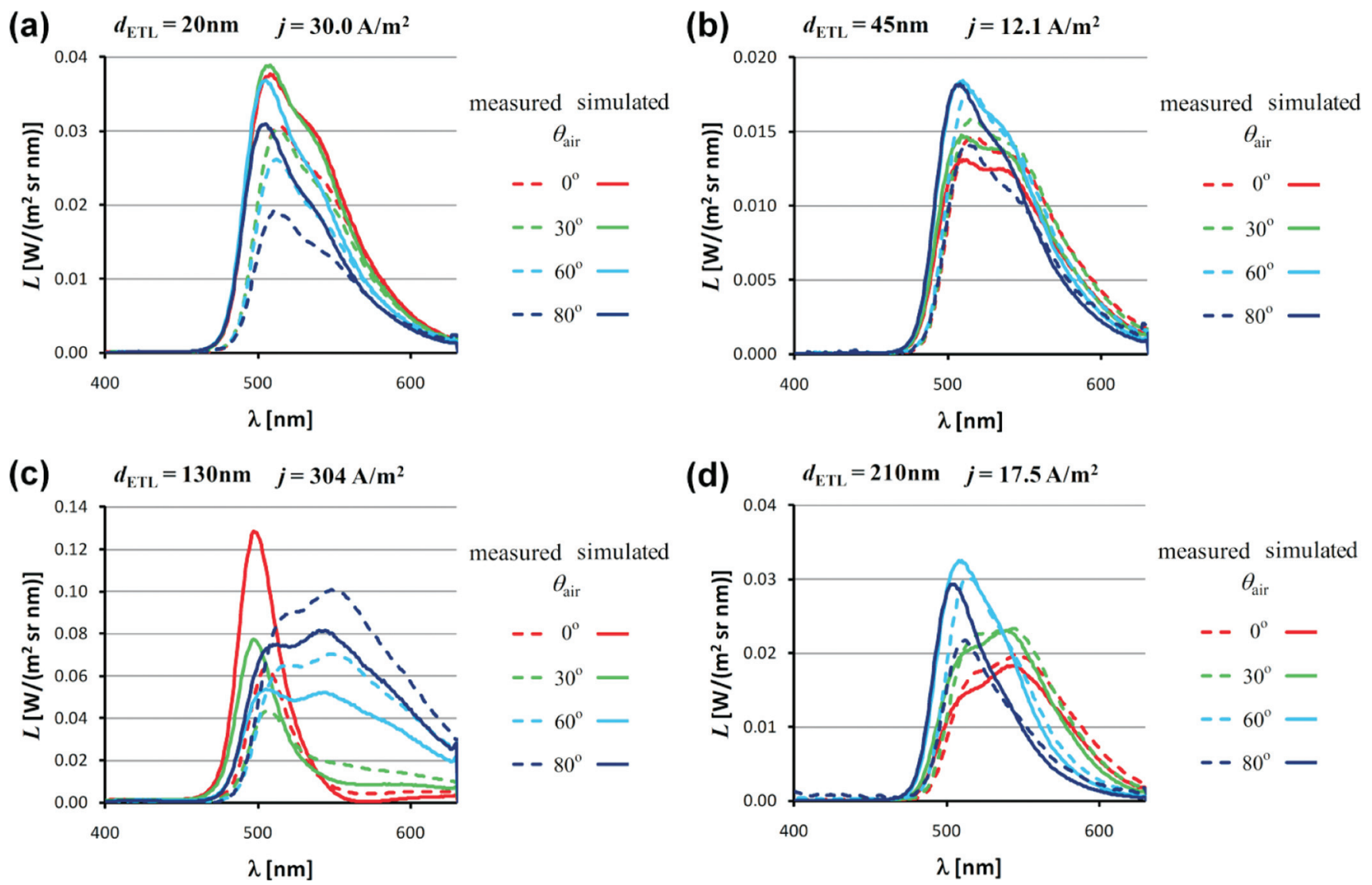

FIG. 9. (Color online) Comparison of simulated (solid lines) and measured (dashed lines) spectral radiance $\mathrm{L}$ as function of the wavelength $\lambda$ and inclination angle $\theta_{\text {air }}$.

measurements are carried out, are shown on Fig. 9. One can see that there is a good fit between the measured and simulated values of $L_{a i r}(\theta, \lambda, j)$. The discrepancy, in particularly seen for the sample with $d_{\mathrm{ETL}}=130 \mathrm{~nm}$, is caused mainly due to the limited accuracy in the thickness of the organic layers by which they are deposited. Namely, since this particular OLED is in the minimum of the EQE, even small changes in the layer thicknesses of 5-10\% can make an important relative change of the $\mathrm{EQE}$ of the device and a strong change in the angular distribution of the emitted light i.e., the power per unit solid angle $P_{\text {air }}\left(\theta_{\text {air }}, \lambda\right)$. Furthermore the higher current density used for these measurements leads to an efficiency roll-off (Fig. 5). In order to confirm this, we simulate the spectral radiance of this OLED using a fitted $d_{\mathrm{ETL}}=123 \mathrm{~nm}$. From the comparison of the simulated and measured $L_{\text {air }}(\theta, \lambda, j)$ (Fig. 10), one can see there is a good fit between the two, which proves our statement above.

Finally we verify the theory for the LCE. In particular, using the previously determined charge balance $\eta_{c b}=0.80$ and decay rates $\Gamma_{r, 0}=0.975 \mu \mathrm{s}^{-1}$ and $\Gamma_{n r, 0}=0.234 \mu \mathrm{s}^{-1}$ we simulate the LCE in air and in the substrate according to Eqs. (24) and (25) as a function of $d_{\text {ETL }}$ (see Fig. 11). These simulations are compared with the measured LCE of our OLEDs without and with an attached half-ball lens. One can see that there is a very good fit between simulations of $L C E_{\text {air }}$ and the measurements without a half-ball lens. However the measurements with a half-ball lens show somewhat smaller values than what is simulated as $L C E_{\text {sub }}$. The main reason for this is same as the measurements of the EQE with a half-ball lens (Fig. 8), i.e., it is rather difficult to completely outcouple the light emitted at higher inclination angles in the substrate $\left(\theta_{\text {sub }}\right)$, if the attached half-ball lens is not big enough. As a confirmation for this we show the $L C E_{\text {sub }}$ by integrating over only a part of the whole interval for the inclination angle i.e., $0<\theta_{\text {sub }}<\pi / 3$ (instead of $0<$ $\theta_{\text {sub }}<\pi / 2$ ). These simulations show a good fit with the measurements with a half-ball lens, proving that the light emitted at higher inclination angles in the substrate is not very well

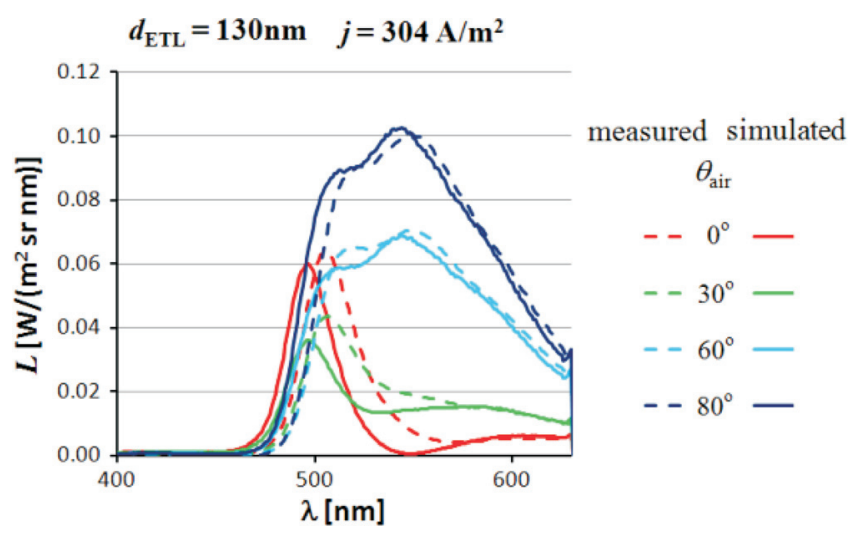

FIG. 10. (Color online) Comparison of measured spectral radiance $L$ for the OLED with $\mathrm{d}_{\mathrm{ETL}}=130 \mathrm{~nm}$ and simulated $\mathrm{L}$ using a fitted $\mathrm{d}_{\mathrm{ETL}}=123 \mathrm{~nm}$. 


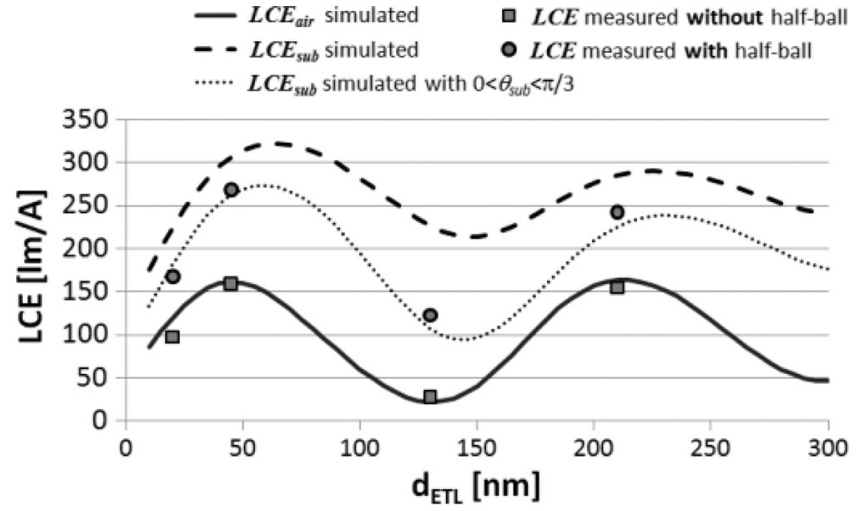

FIG. 11. Simulated luminous current efficacy in air and substrate $\left(L C E_{\text {air }}\right.$ and $L C E_{\mathrm{sub}}$ ) as function of the electron-transport layer thickness $d_{\mathrm{ETL}}$ (solid and dashed line). Simulated $L C E_{\text {sub }}$ by integrating the inclination angle only in the range $0<\theta_{\text {sub }}<\pi / 3$ (dotted line). Measured LCE without and with an attached half-ball lens on the fabricated OLEDs (squares and circles).

outcoupled in the measurements. However, the overall theory for the LCE gives a very good estimation for what is seen in experiments.

\section{CONCLUSIONS}

With this work, we introduce a new integrated model for the light emission from OLEDs with phosphorescent emitters, which takes into account all optical effects present in these devices. In particular, the model includes the exciton decay time change caused by the optical environment and light outcoupling from OLED to substrate and substrate to air. Furthermore, we present a theory on how to calculate the external quantum efficiency, the spectral radiance and the luminous current efficacy of OLEDs. The theory of the integrated model is experimentally verified by a range of measurements on green OLED samples that include: exciton decay time, current voltage-characteristics, external quantum efficiency, spectral radiance and luminous current efficacy. Through a detailed analysis of both, theory and experiments, we estimate the charge balance of these OLEDs, the radiative and nonradiative decay rates of the green $\operatorname{Ir}(\mathrm{ppy})_{3}$ emitter. A challenge for our future work is to develop an integrated model for the different white OLED stacks known today.

\section{ACKNOWLEDGMENTS}

The work leading to these results has received funding from the European Community's Seventh Framework Programme [Grant No.n FP7-224122 (OLED100.eu)]. Part of this work has been supported by the Belgian IAP project photonics@be.

${ }^{1}$ S. Reineke, K. Walzer, and K. Leo, Phys. Rev. B 75, 125328 (2007).

${ }^{2}$ N. K. Patel, S. Cina, and J. H. Burroughes, IEEE J. Sel. Top. Quantum Electron. 8, 346 (2002).

${ }^{3}$ S. Mladenovski, S. Reineke, and K. Neyts, 29th International Display Research Conference (Eurodisplay 2009), Rome, Italy 14-17, Sept. 2009, Galaad Edizioni, Teramo, Italy, pp. 43-46.

${ }^{4}$ L. H. Smith, J. A. E. Wasey, I. D. W. Samuel, and W. L. Barnes, Adv. Funct. Mater. 15, 1839 (2005).

${ }^{5}$ J.-S. Kim, P. K. H. Ho, N. C. Greenham, and R. H. Friend, J. Appl. Phys. 88, 1073 (2000).

${ }^{6}$ K. Neyts, J. Opt. Soc. Am. A 15, 962 (1998).

${ }^{7}$ K. Neyts, Appl. Surf. Sci. 244, 517 (2005).

${ }^{8}$ H. Riel, S. Karg, T. Beirlein, W. Rieß, and K. Neyts, J. Appl. Phys. 98, 5290 (2003)

${ }^{9}$ S. Mladenovski, K. Neyts, D. Pavicic, A. Werner, and C. Rothe, Opt. Express 17, 7562 (2009).

${ }^{10}$ L. Penninck, S. Mladenovski, and K. Neyts, J. Opt. 12, 075001 (2010).

${ }^{11}$ H. Riel, S. Karg, T. Beierlein, B. Ruhstaller, and W. Rieß, Appl. Phys. Lett. 82, 466 (2003).

${ }^{12}$ S. Mladenovski, S. Reineke, and K. Neyts, Opt. Lett. 34, 1375 (2009).

${ }^{13}$ E. M. Purcell, Phys. Rev. 69, 681 (1946).

${ }^{14}$ S. Mladenovski, S. Reineke, L. Penninck, and K. Neyts, J. Soc. Inf. Disp. 19, 80 (2011).

${ }^{15}$ J. Blochwitz, M. Pfeiffer, T. Fritz, and K. Leo, Appl. Phys. Lett. 73, 729 (1998).

${ }^{16}$ G. He, O. Schneider, D. Qin, X. Zhou, M. Pfeiffer, and K. Leo, J. Appl. Phys. 95, 5773 (2004).

${ }^{17}$ S. Reineke, F. Lindner, Q. Huang, G. Schwartz, K. Walzer, and K. Leo, Phys. Status Solidi B 245, 804 (2008).

${ }^{18}$ S. Reineke, F. Lindner, G. Schwartz, N. Seidler, K. Walzer, B. Lussem, and K. Leo, Nature 459, 234 (2009).

${ }^{19}$ C. Hosokawa, H. Tokailin, H. Higashi, and T. Kusumoto, Appl. Phys. Lett. 63, 1322 (1993).

${ }^{20}$ D. Tanaka, Y. Agata, T. Takeda, S. Watanabe, and J. Kido, Jpn. J. Appl. Phys., Part 2 46, L117 (2007).

${ }^{21}$ R. Meerheim, R. Nitsche, and K. Leo, Appl. Phys. Lett. 93, 043310 (2008). 